\title{
Emotion Regulation via the Autonomic Nervous System in Children with Attention-Deficit/Hyperactivity Disorder (ADHD)
}

\author{
Erica D. Musser, \\ Psychology Department, University of Oregon, 1227 University of Oregon, Eugene, OR 97403, \\ USA
}

Psychology Department, Oregon Health \& Science University, 3181 SW Sam Jackson Park

Road, Portland, OR 97239, USA

Department of Psychology, Oregon Health \& Science University, 3181 SW Sam Jackson Park Road, 1506/UHN-80R1, Portland, OR 97239, USA

Richard W. Backs,

Psychology Department, Central Michigan University, 1200 North Franklin Street, Mount

Pleasant, MI 48859, USA

Colleen F. Schmitt,

Psychology Department, Oregon Health \& Science University, 3181 SW Sam Jackson Park

Road, Portland, OR 97239, USA

Jennifer C. Ablow,

Psychology Department, University of Oregon, 1227 University of Oregon, Eugene, OR 97403, USA

Jeffery R. Measelle, and

Psychology Department, University of Oregon, 1227 University of Oregon, Eugene, OR 97403, USA

Joel T. Nigg

Psychology Department, Oregon Health \& Science University, 3181 SW Sam Jackson Park

Road, Portland, OR 97239, USA

Erica D. Musser: mussere@ohsu.edu

\section{Abstract}

Despite growing interest in conceptualizing ADHD as involving disrupted emotion regulation, few studies have examined the physiological mechanisms related to emotion regulation in children with this disorder. This study examined parasympathetic and sympathetic nervous system reactivity via measures of respiratory sinus arrhythmia (RSA) and cardiac pre-ejection period (PEP) in children with ADHD $(n=32)$ and typically developing controls $(n=34)$, using a novel emotion task with four conditions: negative induction, negative suppression, positive induction, and positive suppression of affect. Both groups showed strong task-response effects in RSA. However, typically developing children showed systematic variation in parasympathetic activity (RSA) depending on both emotion valence (more activation for negative emotion, reduced activation for positive emotion) and task demand (more activation for suppression than induction). In contrast, children with ADHD displayed a stable pattern of elevated parasympathetic activity (RSA) across all task conditions compared to baseline. No group differences in sympathetic

(C) Springer Science+Business Media, LLC 2011

Correspondence to: Erica D. Musser, mussere@ohsu . edu. 
activity (PEP) were observed. It is concluded ADHD in childhood is associated with abnormal parasympathetic mechanisms involved in emotion regulation.

\section{Keywords}

Attention-deficit/hyperactivity disorder (ADHD); Autonomic reactivity; Emotion regulation

Attention-deficit/hyperactivity disorder (ADHD) has been previously theorized to involve dysfunctions in the physiological and neural systems supporting behavioral inhibition and control (Barkley 1997; Nigg 2001), with an emphasis on inability to regulate behavior. Yet, emotional dysregulation has long been seen as part of the condition. For example, Wender's work in the 1970s pointed to the role of emotion dysregulation in ADHD, as he described that that adults with ADHD are characterized by a number of emotional and internalizing problems as well as emotional labiality (reviewed in Wender 1995). However, this work in adults left it unclear whether emotional dysregulation is a secondary symptom present in only some individuals or a core feature by which we may understand the condition. A developmental view of the centrality of regulatory difficulty in ADHD from early childhood has been offered by Nigg et al. (2006). Concomitantly, and more recently with the recognition similar control processes are also involved in emotion and its regulation, interest has crystallized in clarifying the role of emotion regulation in ADHD (Barkley and Fischer 2010; Martel 2009; Wehmeier et al. 2010). Given how critical this idea of emotion dysregulation is for conceptualizing ADHD, it warrants further empirical evaluation.

Additionally, adding specificity to these claims is essential to avoid the development of an excessively broad theory, as emotion regulation in some form is likely related to nearly all forms of psychopathology. For example, if emotion includes both positive (or approach) and negative (or avoidance) systems, it is unclear which system would be most affected in ADHD, though several theories have proposed dysfunction in the positive (approach) system, consistent with elevations in excitability and anger or with lack of motivation or arousal. These theories are based in behavioral research demonstrating disruptions of reward sensitivity in children with ADHD and on the role of the appetitive, dopaminergic systems, including the nucleus accumbens and ascending limbic-frontal dopaminergic networks, in ADHD (for a review see Nigg 2006).

Seeing ADHD through the lens of emotion regulation has been spurred by findings linking temperament with ADHD (Martel and Nigg 2006) and the high level of comorbidity of ADHD with mood disorders (Chronis-Tuscano et al. 2010; Elia et al. 2008), though emotoin dysregulation can also be associated conceptually with anger, tantrums, excitability, and impulsivity. With regard to emotion per se, ADHD has been associated with emotional reactivity and with reduced emotional inhibition, recognition, and empathy (Cadesky et al. 2000; Maedgen and Carlson 2000; Walcott and Landau 2004). Research in this domain has begun to suggest that emotional impulsiveness is a central feature of ADHD, and emotional impulsiveness has been shown to be associated with impairment over and above that associated with the traditional dimensions of inattention, hyperactivity, and behavioral impulsivity (Barkley and Fischer 2010). However, few studies have examined directly emotion regulation in ADHD (for an exception see Melnick and Hinshaw 2000). Almost none have done so using autonomic nervous system functioning as a physiological index of emotion regulation under emotion induction and suppression conditions, thus leaving it unclear whether regulation is impaired in ADHD per se, and not merely in comorbid individuals. 
Emotion regulation is conceptualized herein as described by Gross $(1998,2007)$. According to this framework, emotions are biologically based reactions and are brief and malleable, resulting in changes in physiology, subjective experience, and expressive behavior. Emotion regulation is the manipulation of the physiological, subjective, or behavioral components of the emotional response. Suppression is one type of emotion regulation that involves consciously inhibiting one's expression behaviors during emotional arousal (Gross and Levenson 1997; Gross 1998; Gross and Levenson 1993). It has the attractive feature of being readily manipulated in the laboratory. In typically developing adults, suppression influences subjective ratings of emotion, expressive behavior, physiological reactivity, and neural responding (Crowell et al. 2006; Goldin et al. 2008; Gross and Levenson 1993). Methodologically, a successful approach has been to induce emotional arousal via clips from emotionally evocative films. Participants are instructed to exhibit (or enhance) the emotion displayed in the film and/ or to suppress it.

Related physiological indices have been developed on the basis of a substantive theoretical and empirical literature (Berntson et al. 1997; Porges 1995). Parasympathetic-linked cardiac activity is indexed by respiratory sinus arrhythmia (RSA), which has been associated with emotion regulation (Beauchaine 2001; Berntson et al. 1997). RSA provides a relatively pure index of parasympathetic control of the heart through the innervations of the vagus nerve (Berntson et al. 1993b). RSA has good long-term temporal consistency (Berntson et al. 1993a), and predicts vagal control during pharmacological blockade. Similarly, sympatheticlinked cardiac reactivity has been indexed by cardiac pre-ejection period (PEP), which has been associated with emotional arousal, mental effort, insensitivity to reward, and in some contexts, approach and avoidance behaviors (Beauchaine 2001; Berntson et al. 1997; Kelsey et al. 2007). PEP is a commonly used index of sympathetic cardiac control of the heart through the beta-adrenal system (Berntson et al. 1994). PEP represents the time between the depolarization of the left ventrical of the heart and the onset of ejection of blood into the aorta. Shorter PEP values have been associated with increased sympathetic activation. PEP has satisfactory long-term temporal consistency (Burleson et al. 2003). Individual differences in PEP have been predictive of cardiac sympathetic and beta-adrenal control as indexed by pharmacological blockades (Cacioppo et al. 1994).

These physiological indices have been used to examine emotion regulation in the context of externalizing behaviors in children, but to a lesser degree in ADHD. This is particularly important because it is not clear that ADHD and externalizing problems are the same in this regard, despite their common behavioral co-occurrence. Specifically, in children, autonomic dysregulation at rest has been associated with aggression (Mezzacappa et al. 1997), inattention (Suess et al. 1994), behavioral inhibition (Kagan and Snidman 1991), decreased empathy (Fabes et al. 1994), emotion labiality (Beauchaine 2001), hostility (Sloan et al. 1994), and temperamental reactivity (Calkins 1997). Boys with externalizing symptoms tend to have lower baseline heart rate and RSA than controls (Mezzacappa et al. 1997). Furthermore, in a recent series of studies by El-Sheikh et al. (2009) it was reported that opposing action of the PNS (indexed by RSA reactivity) and SNS (indexed by skin conductance reactivity) in the form of coactivation or augmentation from baseline levels of functioning, acted as a risk factor for the development of externalizing behaviors in environments characterized by inter-parental conflict. However, the coordination of these systems, that is, reciprocal PNS and SNS activation, served as a protective factor.

Although the minority of these studies examined physiological reactivity during emotion induction or challenge tasks, results from Beauchaine et al. (2007) and Calkins (1997) suggest that child externalizing behavior is related to inflexible parasympathetic responding during negative emotion induction and reduced sympathetic nervous system responding to rewards. However, neither study used an induction/suppression paradigm, which leaves it 
unclear to what extent the differences in physiological reactivity are a direct result of emotion regulation.

Thus, the association between altered autonomic functioning at baseline and externalizing behavior is rather well established, but the functioning of autonomic regulatory response is less well understood, particularly for ADHD. Indeed, only five studies have examined emotion induction using physiological methods in relation to child externalizing behavior (Boyce et al. 2001; Eisenberg et al. 1997; Herpertz et al. 2005; Marsh et al. 2008; Mead et al. 2004) and none that did so with ADHD (or using emotion suppression paradigms to challenge regulatory capacities). These studies generally show that children with externalizing disorders tend to respond with lower levels of autonomic reactivity in both branches during emotion induction. However, it remains unclear to what extent the conclusions drawn about externalizing behavior will apply to ADHD when comorbid externalizing behavior is accounted for.

In this regard, Beauchaine et al. (2001) found that compared with typically developing children, both adolescents with ADHD and with conduct disorder exhibited reduced skin conductance (sympathetic nervous system responding) during a variable reward task, but nearly all remaining autonomic nervous system effects were confined to the conduct disorder group. In pre-school aged children, Crowell et al. (2006) found that both ADHD and oppositional disorder were characterized by reduced electrodermal responding and lengthened PEP (decreased sympathetic functioning) at baseline, and heart rate changes in response to a reward task in these groups were mediated exclusively by parasympathetic withdrawal, unlike controls. These results suggested similar profiles of parasympathetic functioning for the ADHD and oppositional groups, but remain preliminary and in the context of reward tasks, rather than tasks requiring the regulation of emotion. They also omit the primary age range for ADHD identification, the early school age years.

In conclusion, there is need to clarify the nature of autonomic activity during emotion regulation in children with ADHD and to extend this literature to emotional challenge tasks, like suppression. We hypothesized that ADHD would be related to alterations in response to emotion induction and suppression in both the parasympathetic and sympathetic systems. Specifically, we hypothesized that children with ADHD will have a pattern of autonomic reactivity that is minimally augmented from homeostatic functioning across the changing affective and regulatory demands of the induction and suppression task. This would be consistent with clinical impression that children with ADHD fail to adapt their emotional reactivity to contextual demands. However, we hypothesized that typically developing children will show autonomic flexibility in the regulation of emotion as the task demands change, that is, increased RSA with increased regulatory demand during suppression. It is hypothesized that these differences will be particularly prominent when attempting to regulate positive affect, due to theories suggesting alterations in approach-related neural systems in ADHD (Nigg 2006; Nigg et al. 2004).

\section{Methods}

\section{Participants}

Participants were 66 children aged 7 to 9 years; 32 met DSM-IV criteria for ADHD (19 combined type, 13 inattentive type), and 34 were non-ADHD comparison youth (American Psychiatric Association, 2000). The middle childhood age range was selected as ideal to test the hypotheses in the literature, as this is the age of peak identification of ADHD, the period when new demands for regulation are put upon children with ADHD due to school, tests the theory that regulation problems are detected early in the developmental course, and avoids confounds of adolescence and associated emergence of depression, substance use, and 
extended medication treatment, while still having children old enough for self regulation to be demanded by their surroundings. Thus, we deliberately restricted the study to this age range. Families were recruited through advertisements and mailings in order to obtain a broadly representative sample that would not be biased by clinic referral or identification. The local Institutional Review Board approved the study; all procedures conformed to the Ethical Principles of Psychologists and Code of Conduct (American Psychological Association 2002). Parents provided written informed consent and children provided written assent for all procedures.

Recruitment and Identification-Families volunteering for the study were passed through a multi-gate screening process to establish eligibility and diagnostic group assignment. At stage one, parents of potential participants completed a phone interview to rule out use of ineligible medications (including all non-stimulant, or long acting psychoactive medications that could not be washed out for the study), neurological impairments, history of seizures, traumatic brain injury, major medical conditions, or mental retardation or pervasive developmental disorders.

At stage two, a parent and a teacher of remaining eligible youth completed the ADHD Rating Scale (DuPaul et al. 1998), Conner's Rating Scale-3rd Edition (Conners 2008), and the Strengths and Difficulties Questionnaire (Goodman 2001). A parent also was interviewed with the Kiddie Schedule for Affective Disorders and Schizophrenia (KSADSE; Puig-Antich and Ryan 1996) by a trained master-degree level clinician. The semistructured interviews were monitored for fidelity and evaluated for inter-interviewer reliability, $k>0.80$ for ADHD for all interviewers. Children completed an IQ screening consisting of the Block Design, Vocabulary, and Information sub-tests from the Wechsler Intelligence Scales for Children, Fourth Edition (WISC-IV; Wechsler 2003). Children also completed the Word Reading, Math Reasoning, and PseudoWord subscales from the Wechsler Individual Achievement Test, Second Edition (WIAT-II; Wechsler 2005) to enable evaluation of learning disability and academic impairment.

Final ADHD and Other Diagnoses-Results from the KSADS-E, rating scales, and child IQ and achievement tests were presented to a diagnostic team (a board certified child psychiatrist and a licensed neuropsychologist) each of whom arrived independently at a "best estimate" diagnosis for ADHD and subtype and all other disorders assessed by the KSADS-E, using DSM-IV criteria. Their agreement rates were acceptable, $k>0.80$ for all disorders with a $>5 \%$ base rate in the sample. Disagreements were resolved by conference. If consensus was not easily achieved, the case was excluded. This occurred in five cases.

A diagnosis of ADHD required that the child's symptoms had a cross-situational presentation, evidence of impairment, and were better not accounted for by another disorder, as specified in DSM-IV. If both parent and teacher provided standardized ratings of at least the 90th percentile on a rating of inattention or hyperactivity using the above-named rating scales, then symptoms were counted as present if endorsed by the parent on the KSADS-E or the teacher on the ADHD rating scale. The teacher was allowed to contribute a maximum of two additional symptoms (that is, at least four symptoms had to be identified on the KSADS). This procedure was similar to that used in the DSM-IV field trials and the MTA study. The "or" algorithm was only used in roughly $6 \%$ of cases for which there was disagreement between the teacher ratings and adult ratings. Specifically, teachers rated 30 children as having six symptoms, while parents rated 32 children as having ADHD. Thus, thirty of thirty-two children met criteria according to both the teacher and the parent.

Exclusion criteria included an estimated Full Scale IQ < 75, major depressive disorder, lifetime or current mania or psychosis, or learning disability. Other disorders were free to 
vary. A total of 25 children were excluded from the study due to comorbid disorders (20) or lack of agreement by the diagnostic team (5).

Medication Washout-All children were required to be medication free at the time of testing. Therefore, prior to the task, children taking stimulant medication underwent a $24-48$ $\mathrm{h}$ washout, dependent on the type of stimulant preparation they were prescribed. Seven ADHD children (and no controls) were prescribed stimulants (one amphetamine/ dextroamphetamine; two dexmethylphenidate; two methylphenidate; two methylphenidate HCL). As an extra precaution, we covaried stimulant prescription status (present, absent) to remove the effect of the med washout, with no effect on results. Thus, we can be confident that results are not related to medication effects or to medication washout effects.

Emotion Induction Procedure-Each child underwent the emotion induction and suppression procedure using both a negative and a positive emotion-laden film clips. This involved watching four, 2-min film clips taken from Homeward Bound, a film about dogs and a cat who are separated and reunited with their human family.

The first two clips were presumed to elicit negative emotions, while last two segments were presumed to elicit positive emotions. To evaluate the validity of these conditions, children completed the Self-Assessment Manikin (SAM) valence and arousal scales (Bradley and Lang 1994) for each clip. The SAM is a pictorial assessment, which measures self-reported valence and arousal on 5-point scales.

In the induction condition, children were asked to facially mimic the emotion of the main character. This instruction was given for the first negative and first positive segment. In the suppression condition, the child was to imagine what the main character was feeling, but to keep his or her face still, masking (suppressing) the emotion. This instruction was given for the second negative and second positive segment. These conditions were not counterbalanced as: (a) it was important to end with positive emotion for human subjects' welfare, (b) the film was a continuous story, and changing the order would have confounded suppression with cognitive challenge to interpret the story, (c) putting induction prior to suppression maximized the suppression challenge. Thus, the same sequence of the four task conditions was presented to each child: (1) negative induction, (2) negative suppression, (3) positive induction, and (4) positive suppression.

A resting baseline of 2 min was presented before the task, and neutral baseline periods of 2 min were presented before the negative task condition and before the positive condition. Each neutral baseline consisted of observing a set of ten neutral pictures from the International Affective Picture System (IAPS; Lang et al. 1999). This type of baseline accounts for the physiological response of orienting and attending (Jennings et al. 1998). The SAM was also used to assess self-reported valence and arousal during these baselines.

\section{Physiological Recording}

Overview-Disposable silver/silver-chloride electrodes were placed in an electrocardiogram (ECG) and impedance cardiography (ICG) configuration, as described below. The ECG electrodes were placed at the right collar bone and the tenth-left rib with a ground electrode placed at the tenth-right rib. For ICG, two voltage electrodes were placed below the suprasternal notch and xiphoid process, and two current electrodes were placed on the back 3 to $4 \mathrm{~cm}$ above and below the voltage electrodes. ECG and ICG were recorded throughout each of the baselines and task epochs. The R-R series was sampled at 1,000 Hz. Interbeat-interval (IBI) and respiration rate (RR) data were derived using the ECG and ICG data. 
Cardiac Pre-ejection Period (PEP)—PEP was derived from ECG and ICG, using $60 \mathrm{~s}$ epochs, using MindWare Impedance Cardiography V. 2.6 (MindWare 2008b), allowing for simultaneous editing of the data obtained from ECG and ICG. PEP was indexed as the time interval in milliseconds from the onset of the Q-wave to the B point of the $\mathrm{dZ} / \mathrm{dt}$ wave, using the method outlined by (Berntson et al. 2004). Artifacts were examined and removed using the software and visual inspection completed by two raters, $k>0.89$ for each epoch. There were no between-group differences in the rate of artifacts, all $p>0.5$.

Respiratory Sinus Arrhythmia (RSA)—RSA was indexed by extracting the high frequency component $(>0.15 \mathrm{~Hz})$ of the R-R peak time series. R-R waves were examined for artifacts and outliers using MindWare ${ }^{\circledR}$ Heart Rate Variability software V. 2.6 (MindWare 2008a). Artifacts were removed using the software and visual inspection completed by two raters for validity, all $k>0.93$. There were no between-group differences in the rate of artifacts, all $p>0.5$.

RSA was derived using spectral analysis (Berntson et al. 1997), in $60 \mathrm{~s}$ epochs. Spectral analysis was performed on the R-R time series from the ECG (Berntson et al. 1997). The time series was detrended and submitted to a Fourier transformation. The high frequency band $\left(\ln \left(\mathrm{ms}^{2}\right)\right)$ was set over the respiratory frequency band of 0.24 to $1.040 \mathrm{~Hz}$. Respiratory rates and amplitudes were derived from the impedance cardiograph signal (Z0) ensuring that these signals remained within the analytical bandwidth.

\section{Results}

\section{Preliminary Analyses}

Power Analysis-G*Power was used to determine the power to detect effects with our sample size using a repeated measures ANOVA (Faul et al. 2007). Using a sample size of 66 participants, power was 0.80 to detect an ADHD vs. non-ADHD group difference, $p<0.05$, of $d=0.6$. Similarly, with a total sample size of 66 participants, in repeated measures analyses, power was 0.80 to detect condition effects (valence effects, regulation effects), and to detect group by condition (valence and regulation demand) interaction, $p<0.05$, of $d=0.3$, which is a small to medium effect size (Cohen 1992). Adding covariates (e.g., gender, the use of stimulant medications, comorbid disorders) reduces this power estimate only slightly. Thus, power was adequate to detect the size of effects typically of interest in ADHD related research, which range above $d=0.5$ for group effects (Willcutt et al. 2005).

Descriptive and Diagnostic Statistics-Descriptive statistics are reported by group in Table 1. Groups did not differ with respect to age, family income, race, or IQ. Inclusion of these variables did not affect any of the main analyses, so these variables were excluded and are not discussed further. Groups differed with respect to gender, so it was covaried in all analyses. Its inclusion did not affect any of the results reported; all results are shown with gender covaried. Clinical characteristics are also provided Table 1. The ADHD and control groups differed significantly in terms of both parent and teacher rated Inattention and Hyperactive/ Impulsive symptom T-scores on the Conner's 3rd Edition and in terms of both parent and teacher rated symptoms counts on the ADHD-RS, all $F>42$ (see Table 1). Comorbid diagnostic statistics are also presented in Table 1. Groups did not differ with respect to the frequency of current anxiety, mood, oppositional-defiant, conduct, or tic disorders, all $\mathrm{F}<1.89$. The low rate of comorbidity in this sample reflects the caution with which we ruled out children whose ADHD might be explained by a comorbid disorder (per DSM-IV). The inclusion of comorbid disorders as covariates (individually or collectively) did not affect any of the main study results, and these effects are not further discussed. Results are shown with the total number of comorbid disorders covaried. 
Baseline Effects-It was important to show that there was stability in the physiology measures across baseline conditions to confirm that these were stable periods of rest. The mean scores of RSA, PEP, IBI, and RR were calculated for the first and second 60-s epochs of both the resting and the two neutral pictures baselines. None of the physiology parameters assessed differed statistically from the first minute to the second, all $F<1.0, p>0.50$. Only the second baseline epoch $(60 \mathrm{~s})$ is used in subsequent analyses and calculations of change scores to account for any effect of recovery.

Groups did not differ significantly in resting or neutral picture baseline activity across any of the physiological parameters, all $F<1.0$, and groups did not differ in their SAM ratings of the neutral pictures, all $F<1.0$. Thus, any differences in autonomic reactivity during the tasks could not be attributed to differences in baseline autonomic functioning or mood prior to the task.

Effectiveness of Emotion Induction by Self-report-A $2 \times 2 \times 2$ repeated-measures ANOVA (valence[negative/positive] $\times$ regulation[induction/suppression] $\times$ group[control/ ADHD]) was used to assess SAM (a) valence and (b) arousal scores across each of the four task epochs. Eta ${ }^{2}$ was included as a measure of effect size as is typically used in ANOVA. As a rule of thumb an eta ${ }^{2}$ of 0.01 is small, 0.06 is medium, and 0.14 is large (Cohen 1992). The results of the repeated-measures ANOVA supported the validity of the positivenegative emotion induction and suppression manipulations.

Beginning with valence, scores differed according to both the valence condition of the task, $\mathrm{F}(1,64)=214.1, p<0.001$ e eta $^{2}=0.77$, and the regulation condition, $\mathrm{F}(1,64)=9.97, p<0.001$, eta $^{2}=0.135$. The interaction of valence and regulation conditions was significant, consistent with the interpretation that the experience of each emotional valence (negative and positive) was greater in the suppression than the expression of an emotion, particularly for negative emotions, $\mathrm{F}(1,64)=26.752, p<0.001$, eta $^{2}=0.30$, which is consistent with previous (Gross and Levenson 1993). Analysis of the simple effects confirmed that the four conditions differed as indicated by our labeling of the conditions: higher (more positive) ratings for positive than negative emotion, all $p<0.001$; higher (more positive) ratings for induction than suppression, all $p<0.001$, see Table 2 . Also reassuringly, the ADHD and control groups were similar with respect to SAM valence rating scores, as none of the interactions involving group status were significant, all $F<1.0$, suggesting that interpretation of changes in heart rate variability (IBI) were not confounded by differences in self-reported valence appraisal of the task conditions.

The arousal rating scores showed a convergent picture. There was a significant main effect for arousal scores between positive and negative segments, $\mathrm{F}(1,64)=19.03, p<0.001$, eta $^{2}=0.23$, although only a trend for suppression versus induction, $\mathrm{F}(1,64)=3.118, p<0.08$, eta $^{2}=0.05$. Again, none of the interactions involving group status were meaningful, all $F<1.0$. Thus, on the whole these data suggest that the task manipulations were effective and valid across both groups.

Task Habituation and Order Effects-Repeated measures ANOVA indicated that none of the physiological parameters differed across the neutral pictures baselines, all $F<1.0$ for RSA and PEP, suggesting no systematic variations based solely on time. Second, a polynomial repeated measures ANOVA for the full sample revealed that the linear effect of time on RSA across all conditions was nonsignificant, $\mathrm{F}(1,33)=2.268, p=0.142$, as was the quadratic effect, $\mathrm{F}(33)=1.469, p=0.234$, whereas the cubic effect was significant, $\mathrm{F}(1$, $65)=13.461, p<0.001$, eta $^{2}=0.18$. This is consistent with participants responding to the task manipulations and inconsistent with a habituation effect. Simple examination of the means (Table 3; Fig. 1) confirms that RSA did not simply decrease across time, and a similar 
pattern was observed for PEP and each of the other physiological parameters measured for both the linear effects, all $F<1.0, p>0.15$, as well as the quadratic effect, all $F<1.0, p>0.25$. We conclude that habituation and order effects were trivial relative to task manipulation effects.

\section{Effects of Emotion Induction and Suppression on RSA and PEP}

Overall Effects on PEP- $2 \times 2 \times 2$ repeated-measures ANOVA examined the effects of task condition on reactivity change scores for PEP. The main effect of both the valence and regulation conditions on PEP were nonsignificant, all $F<1.9, p>0.18$, as were both the interactions by group, all $F<1.0$. The differences between the groups across the task conditions for PEP therefore were not examined further.

Overall Effects on RSA-The means of the RSA reactivity scores for each task epoch are listed in Table 3. $2 \times 2 \times 2$ repeated-measures ANOVA examined the effects of task condition on reactivity change scores for RSA. Both relevant interactions were significant: valence $\times$ group and regulation $\times$ group, $\mathrm{F}(1,64)=9.144, p<0.005$, eta ${ }^{2}=0.13$ and $\mathrm{F}(1$, 64) $=9.121, p<0.005$, eta $^{2}=0.13$, respectively. Therefore, main effects were not interpreted and simple effects were pursued.

Examinations of simple effects revealed a significant difference in RSA reactivity scores between ADHD and control groups during both the negative suppression and positive induction condition (Table 3; Fig. 1). Compared to baseline, during the negative suppression condition, the ADHD group showed a smaller increase than the control group, $\mathrm{F}(1,64)=5.163, p<0.05$ eta $^{2}=0.09$. In the positive induction condition, the ADHD group's RSA increased from baseline, whereas the control group decreased, $\mathrm{F}(1,64)=5.213, p<0.05$, eta $^{2}=0.09$. Covarying age or gender did not change these results.

Follow Up Checks on RSA Results-To determine whether the reactivity changes associated with task epoch were better explained by parasympathetic-based emotion regulation (RSA), rather than changes in other aspects of the participants' physiology (e.g., those due to sympathetic control), the main effects and interactions for IBI and RR were examined using the same $2 \times 2 \times 2$ repeated-measures ANOVA. The main effect of task valence (positive/negative affect) was not significant for any of these parameters, all $F<1.0$. There was a significant main effect of regulation (suppression/induction) for IBI, $\mathrm{F}(1,64)=18.683, p<0.001$, eta $^{2}=0.23$ and $\mathrm{F}(1,64)=17.953, p<0.001$, eta $^{2}=0.22$ with IBI changing in the task-appropriate direction. However, none of the interactions by group were significant, all $F<1.50, p>0.20$. Thus, groups did not differ on general, multidetermined physiological parameters, but only in the specific domain of RSA, indexing PNS functioning.

Finally, presented a different way, repeated-measures ANOVA were used to examine the pattern of the RSA change across the task conditions for each of the groups. For the control group, the omnibus ANOVAwas significant, $\mathrm{F}(1,64)=9.43, p<0.001$, eta ${ }^{2}=0.13$, indicating that there were meaningful changes in RSA across each of the four task epochs. The linear effect was non-significant, $\mathrm{F}(1,33)=2.208, p=0.162$, as was the quadratic effect, $\mathrm{F}(33)=2.051, p=0.142$, whereas the cubic effect of time on RSA effect significant, $\mathrm{F}(1$, $33)=20.033, p<0.001$, eta $^{2}=0.25$, which is consistent with controls responding flexibly to the segment manipulations. Table 3 shows that the RSA reactivity scores were in the appropriate direction (also Fig. 1). For the ADHD group, the omnibus ANOVA was nonsignificant, $\mathrm{F}(1,32)=0.622, p=0.61$, and none of the individual polynomial effects were significant, all $F<1.0$, consistent with ADHD participants responding to the task, but inflexibly so across conditions. 


\section{Discussion}

This study examined emotion regulation and autonomic nervous system functioning in both the parasympathetic (as indexed by RSA) and sympathetic (as indexed by PEP) systems among children with and without ADHD, during induction and suppression of negative and positive emotions. The major hypothesis was confirmed. Children with ADHD displayed an slightly augmented from homeostasis pattern of RSA across the four task conditions compared to typically developing children, consistent with altered physiological functioning in parasympathetically-mediated emotion regulation processes. The hypothesis about sympathetic effects (PEP), however, was not supported.

This conclusion about RSA was supported by several ancillary findings that ruled out alternative interpretations. Neither stimulant medication history nor comorbid diagnoses of any kind were associated with differences in parasympathetic or sympathetic functioning across the task conditions. Autonomic functioning at rest and during emotionally neutral tasks did not differ between ADHD and control children, suggesting that these differences were not due to preexisting differences in homeostatic autonomic functioning or mood. Additionally, autonomic differences among children with and without ADHD were not due to differences in age or gender, which is consistent with previous research with this age group (Beauchaine 2001).

To our knowledge, this study is the first of its kind in children, as it measured autonomic reactivity in both branches (parasympathetic and sympathetic), during emotion induction and suppression in response to negative and positive emotion films. This novel paradigm was helpful in several ways. First, it was determined that this type of task can be utilized with this age group and population. The pattern and magnitude of autonomic activity for the control group was similar to that observed among healthy adults (Gross and Levenson 1997). This is valuable, as it will enable further evaluation of emotion suppression in this young, developing age range.

Second, the measurement of multiple domains of autonomic activity allows for specification of the roles of the different branches of the autonomic system in ADHD and in emotion regulation. Group differences in RSA rather than differences associated with the sympathetic nervous system or other aspects of physiology were observed across the task conditions, suggesting that these differences were a function of the parasympathetic branch. Although previous studies by Beauchaine et al. (2007) and Crowell et al. (2006) have found differences in sympathetic functioning among children with ADHD and conduct problems when compared to typically developing youth, both of these studies used reward-based tasks, which are known to strongly engage the sympathetic system. In the adult literature, similar findings have been reported consistently by Raine (1996), who cited under arousal of both branches of the ANS, as reflected by low resting heart rate; however, this work has been conducted during periods of rest, and this group has shown that the association is most characteristic of aggression, antisocial personality disorder, and criminal behavior (Lahey et al. 1993; Raine et al. 1997). As the task used in the current study focused on the regulation of emotions, the key differences between groups was observed in the parasympathetic branch, which is consistent with similar work conducted in adult populations (Berntson et al. 1993b; Gross and Levenson 1993).

Third, the use of both induction and suppression conditions was novel for this age group and clarified that problems in ADHD are not merely in suppressing emotional expression but in regulating affective experiences more generally. Children in the ADHD group responded to both induction and suppression conditions with inefficient parasympathetic responding. 
Fourth, the use of both negative and positive emotional conditions amplified that conclusion. Children in the ADHD group had ineffective parasympathetic responding in both conditions. However, the effect was most pronounced for the positive induction condition, where ADHD was associated with an increase in RSA from baseline, rather than the decrease seen in typically developing children. This finding is in line with previous work and theory which suggests that ADHD is uniquely associated with disruptions in positive emotion or approach systems (Martel 2009; Nigg 2006). Additionally, as groups did not differ in sympathetic activity, it may be that children with ADHD experienced a normal level of emotional or physiological arousal as difficult to manage, while the typically developing children experienced this level of arousal as congruent, pleasant, or easy to manage. Thus, children with ADHD may be similar to children with other externalizing problems, as they may tend to misinterpret emotional information and have difficulty with empathy (see for review, Trentacosta and Fine 2010). Also of note, the groups differed significantly during the negative suppression condition. Several studies have found higher levels of affective traits such as neuroticism, hostility, and negative emotionality/reactivity among children with ADHD (e.g., Martel and Nigg (2006), for a review see Martel (2009).

Despite the strengths of this study, key limitations and necessary next steps should be noted. First, the sample size was relatively small for detecting moderator effects between groups (e.g., diagnosis $\times$ sex interactions), and too small to evaluate effects of ADHD subtype (e.g., inattentive versus combined). Although the effects reported here cannot be explained by comorbid disorders, future studies may identify even more severe dysregulation in subgroups of children with more severe and comorbid cases of ADHD.

Additionally, the use of generic emotional valence, rather than specific emotions, such as sadness or joy, limits specific conclusions and requires follow up. Despite our validation of condition effects by self-reported valence and arousal associated with each of the task conditions, we cannot fully rule out the possibility that children may have responded based on expectation bias. In future studies, we plan to examine the correspondence of facial expressions across the task conditions with both self-report and physiology data to obtain a fuller picture of emotion regulation among children with ADHD.

Furthermore, the between condition invariance in RSA in the ADHD group might at first seem consistent with the ADHD group being less engaged or less motivated to participate in the task. However, the other physiology data and self-report tend to weigh against this possibility. In particular, both the ADHD and control groups displayed significant changes in RSA from baseline levels of functioning. These changes suggest that the participants were, in fact, attempting to engage with the stimulus materials to a similar degree across groups.

Finally, it will be of interest in larger samples to begin to identify which children with ADHD have this pattern of autonomic dysregulation and whether it is predictive of course, impairment, response to treatment, comorbid disorders, or other clinical outcomes.

In conclusion, this study indicates that children with ADHD display a pattern of inflexible and ineffective physiological responding during emotion regulation. These findings are consistent with hypotheses that ADHD is a disorder that involves alterations of emotion regulation, in addition to difficulties in cognition and behavior. The findings are clinically relevant in that they demonstrate the importance of teaching children with ADHD skills in the areas of emotion recognition and regulation in addition to behavioral and cognitive coping. 


\section{Acknowledgments}

Work on this study was supported by R01 MH59105 (PI: Nigg). The authors would like to thank Theodore Beauchaine, Ph.D. for comments on an early version of this manuscript.

\section{References}

American Psychiatric Association. Diagnostic and statistical manual of mental disorders. 4th ed.. Washington: Author; 2000.

American Psychological Association. Ethical principles of psychologists and code of conduct. American Psychologist. 2002; 57:1060-1073. [PubMed: 12613157]

Barkley, RA. ADHD and the nature of self-control. New York: Guilford; 1997.

Barkley RA, Fischer M. The unique contribution of emotional impulsiveness to impairment in major life activities in hyperactive children as adults. Journal of the American Academy of Child and Adolescent Psychiatry. 2010; 49:503-513. [PubMed: 20431470]

Beauchaine TP. Vagal tone, development, and Gray's motivational theory: toward an integrated model of autonomic nervous system functioning in psychopathology. Development and Psychopathology. 2001; 13:183-214. [PubMed: 11393643]

Beauchaine TP, Katkin ES, Strassberg Z, Snarr J. Disinhibitory psychopathology in male adolescents: discriminating conduct disorder from attention-deficit/hyperactivity disorder through concurrent assessment of multiple autonomic states. Journal of Abnormal Psychology. 2001; 110:610-624. [PubMed: 11727950]

Beauchaine TP, Gatzke-Kopp L, Mead HK. Polyvagal Theory and developmental psychopathology: emotion dysregulation and conduct problems from preschool to adolescence. Biological Psychology. 2007; 74:174-184. [PubMed: 17045726]

Berntson GG, Cacioppo JT, Quigley KS. Cardiac psychophysiology and autonomic space in humans: empirical perspectives and conceptual implications. Psychological Bulletin. 1993a; 114:296-322. [PubMed: 8416034]

Berntson GG, Cacioppo JT, Quigley KS. Respiratory sinus arrhythmia: autonomic origins, physiological mechanisms, and psychophysiological implications. Psychophysiology. 1993b; 30:183-196. [PubMed: 8434081]

Berntson GG, Cacioppo JT, Binkley PF, Uchino BN, Quigley KS, Fieldstone A. Autonomic cardiac control. III. Psychological stress and cardiac response in autonomic space as revealed by pharmacological blockades. Psychophysiology. 1994; 31:599-608. [PubMed: 7846220]

Berntson GG, Bigger JT Jr, Eckberg DL, Grossman P, Kaufmann PG, Malik M, et al. Heart rate variability: origins, methods, and interpretive caveats. Psychophysiology. 1997; 34:623-648. [PubMed: 9401419]

Berntson GG, Lozano DL, Chen YJ, Cacioppo JT. Where to Q in PEP. Psychophysiology. 2004; 41:333-337. [PubMed: 15032999]

Boyce WT, Quas J, Alkon A, Smider NA, Essex MJ, Kupfer DJ. Autonomic reactivity and psychopathology in middle childhood. British Journal of Psychiatry. 2001; 179:144-150. [PubMed: 11483476]

Bradley MM, Lang PJ. Measuring emotion: the self-assessment manikin and the semantic differential. Journal of Behavior Therapy and Expression in Psychiatry. 1994; 25:49-59.

Burleson MH, Poehlmann KM, Hawkley LC, Ernst JM, Berntson GG, Malarkey WB, et al. Neuroendocrine and cardiovascular reactivity to stress in mid-aged and older women: long-term temporal consistency of individual differences. Psychophysiology. 2003; 40:358-369. [PubMed: 12946110]

Cacioppo JT, Berntson GG, Binkley PF, Quigley KS, Uchino BN, Fieldstone A. Autonomic cardiac control. II. Noninvasive indices and basal response as revealed by autonomic blockades. Psychophysiology. 1994; 31:586-598. [PubMed: 7846219]

Cadesky EB, Mota VL, Schachar RJ. Beyond words: how do children with ADHD and/or conduct problems process nonverbal information about affect? Journal of the American Academy of Child and Adolescent Psychiatry. 2000; 39:1160-1167. [PubMed: 10986813] 
Calkins SD. Cardiac vagal tone indices of temperamental reactivity and behavioral regulation in young children. Developmental Psychobiology. 1997; 31:125-135. [PubMed: 9298638]

Chronis-Tuscano A, Molina BSG, Pelham WE, Applegate B, Dahlke A, Overmyer M, et al. Very early predictors of adolescent depression and suicide attempts in children with attention-deficit/ hyperactivity disorder. Archives of General Psychiatry. 2010; 67:1044-1051. [PubMed: 20921120]

Cohen J. A power primer. Psychological Bulletin. 1992; 112:155-159. [PubMed: 19565683]

Conners, KC. Conners 3rd edition manual. New York: Multi-Health Systems, Inc; 2008.

Crowell SE, Beauchaine TP, Gatzke-Kopp L, Sylvers P, Mead H, Chipman-Chacon J. Autonomic correlates of attention-deficit/hyperactivity disorder and oppositional defiant disorder in preschool children. Journal of Abnormal Psychology. 2006; 115:174-178. [PubMed: 16492108]

DuPaul, GJ.; Power, TJ.; Anastopoulos, AD.; Reid, R. ADHD rating scale-IV: Checklists, norms, \& clinical interpretation. New York: Guilford; 1998.

Eisenberg N, Guthrie IK, Fabes RA, Reiser M, Murphy BC, Holgren R, et al. The relations of regulation and emotionality to resiliency and competent social functioning in elementary school children. Child Development. 1997; 68:295-311. [PubMed: 9180003]

Elia J, Ambrosini P, Berrettini W. ADHD characteristics: I. Concurrent co-morbidity patterns in children \& adolescents. Child and Adolescent Psychiatry and Mental Health. 2008; 2:15. [PubMed: 18598351]

El-Sheikh M, Kouros CD, Erath S, Cummings EM, Keller P, Staton L. Marital conflict and children's externalizing behavior: interactions between parasympathetic and sympathetic nervous system activity. Monographs of the Society for Research on Child Development. 2009; 74:1-78.

Fabes RA, Eisenberg N, Karbon M, Troyer D, Switzer G. The relations of children's emotion regulation to their vicarious emotional responses and comforting behaviors. Child Development. 1994; 65:1678-1693. [PubMed: 7859549]

Faul F, Erdrelder E, Lang AG, Buchner A. G*Power 3: a flexible statistical power analysis program for the social, behavioral, and biomedical sciences. Behavior Research Methods. 2007; 112:155159.

Goldin PR, McRae K, Ramel W, Gross JJ. The neural bases of emotion regulation: reappraisal and suppression of negative emotion. Biological Psychiatry. 2008; 63:577-586. [PubMed: 17888411]

Goodman R. Psychometric properties of the strengths and difficulties questionnaire (SDQ). Journal of the American Academy of Child and Adolescent Psychiatry. 2001; 40:1337-1345. [PubMed: 11699809]

Gross JJ. Antecedent- and response-focused emotion regulation: divergent consequences for experience, expression, and physiology. Journal of Personality and Social Psychology. 1998; 74:224-237. [PubMed: 9457784]

Gross, JJ. Handbook of emotion regulation. New York: Guilford; 2007.

Gross JJ, Levenson RW. Emotional suppression: physiology, self-report, and expressive behavior. Journal of Personality and Social Psychology. 1993; 64:970-986. [PubMed: 8326473]

Gross JJ, Levenson RW. Hiding feelings: the acute effects of inhibiting negative and positive emotion. Journal of Abnormal Psychology. 1997; 106:95-103. [PubMed: 9103721]

Herpertz SC, Mueller B, Qunaibi M, Lichterfeld C, Konrad K, Herpertz-Dahlmann B. Response to emotional stimuli in boys with conduct disorder. American Journal of Psychiatry. 2005; 162:11001107. [PubMed: 15930058]

Jennings JR, van der Molen MW, Somsen RJ. Changes in heart beat timing: reactivity, resetting, or perturbation? Biologial Psychology. 1998; 47:227-241.

Kagan J, Snidman N. Temperamental factors in human development. American Psychologist. 1991; 46:856-862. [PubMed: 1928938]

Kelsey RM, Ornduff SR, Alpert BS. Reliability of cardiovascular reactivity to stress: internal consistency. Psychophysiology. 2007; 44:216-225. [PubMed: 17343705]

Lahey BB, Hart E, Pliska S, Applegate B, McBurnett R. Neuropsychological correlates of conduct disorder: a rationale and review of research. Journal of Clinical Child Psychology. 1993; 22:141153. 
Lang, PJ.; Bradley, MM.; Cuthbert, BN. International affective picture system (IAPS): Technical manual and affective ratings. Gainsville: University of Florida; 1999.

Maedgen JW, Carlson CL. Social functioning and emotional regulation in the attention deficit hyperactivity disorder subtypes. Journal of Clinical Child Psychology. 2000; 29:30-42. [PubMed: 10693030]

Marsh P, Beauchaine TP, Williams B. Dissociation of sad facial expressions and autonomic nervous system responding in boys with disruptive behavior disorders. Psychophysiology. 2008; 45:100110. [PubMed: 17868261]

Martel MM. Research review: a new perspective on attention-deficit/hyperactivity disorder: emotion dysregulation and trait models. Journal of Child Psychology and Psychiatry. 2009; 50:1042-1051. [PubMed: 19508495]

Martel MM, Nigg JT. Child ADHD and personality/ temperament traits of reactive and effortful control, resiliency, and emotionality. Journal of Child Psychology and Psychiatry. 2006; 47:11751183. [PubMed: 17076757]

Mead, HK.; Beauchaine, TP.; Brenner, SL.; Crowell, SE.; Gatzke-Kopp, L.; Marsh, P. Autonomic response patterns to reward and negative mood induction among children with conduct disorder, depression, and both psychiatric conditions; Paper presented at the Annual Meeting of the Society for Psychophysiological Research; Santa Fe, NM. 2004.

Melnick SM, Hinshaw SP. Emotion regulation and parenting in AD/HD and comparison boys: linkages with social behavior and peer preference. Journal of Abnormal Child Psychology. 2000; 28:73-86. [PubMed: 10772351]

Mezzacappa E, Tremblay RE, Kindlon D, Saul JP, Arseneault L, Seguin J, et al. Anxiety, antisocial behavior, and heart rate regulation in adolescent males. Journal of Child Psychology and Psychiatry. 1997; 38:457-469. [PubMed: 9232491]

MindWare. Mind ware heart rate variability V 2.6 system. Gahanna: MindWare Technologies; 2008a.

MindWare. Mind ware impedance cardiography V 2.6 system. Gahanna: MindWare Technologies; 2008b.

Nigg JT. Is ADHD a disinhibitory disorder? Psychological Bulletin. 2001; 127:571-598. [PubMed: 11548968]

Nigg JT. Temperament and developmental psychopathology. Journal of Child Psychology and Psychiatry. 2006; 47:395-422. [PubMed: 16492265]

Nigg JT, Goldsmith HH, Sachek J. Temperament and attention-deficit/hyperactivity disorder: the development of a multiple pathway model. Journal of Clinical Child and Adolescent Psychology. 2004; 33:42-53. [PubMed: 15028540]

Nigg, JT.; Hinshaw, SP.; Huang-Pollack, C. Disorders of attention and impulse regulation. In: Cicchetti, D.; Cohen, D., editors. Developmental psychopathology. 2nd ed.. New York: Wiley; 2006. p. 358-403.

Porges SW. Orienting in a defensive world: mammalian modifications of our evolutionary heritage. A Polyvagal Theory. Psychophysiology. 1995; 32:301-318. [PubMed: 7652107]

Puig-Antich, J.; Ryan, N. Kiddie schedule for affective disorders and schizophrenia. Pittsburgh: Western Psychiatric Institute; 1996.

Raine, A., editor. Autonomic nervous system activity and violence. Hillsdale: Erlbaum; 1996.

Raine A, Venables R, Mednick S. Low resting heart rate at age three years predisposes to aggression at age 11 years: evidence from the Mauritius Child Health Proejct. Journal of the American Academy of Child \& Adolescent Psychiatry. 1997; 36:1457-1464. [PubMed: 9334560]

Sloan RP, Shapiro PA, Bigger JT Jr, Bagiella E, Steinman RC, Gorman JM. Cardiac autonomic control and hostility in healthy subjects. American Journal of Cardiology. 1994; 74:298-300. [PubMed: 8037145]

Suess PE, Porges SW, Plude DJ. Cardiac vagal tone and sustained attention in school-age children. sychophysiology. 1994; 31:17-22.

Trentacosta CJ, Fine SE. Emotion knowledge, social competence, and behaivor problems in childhood and adolescence: a meta-analytic review. Social Development. 2010; 19:1-29. [PubMed: 21072259] 
Walcott CM, Landau S. The relation between disinhibition and emotion regulation in boys with attention deficit hyperactivity disorder. Journal of Clinical Child and Adolescent Psychology. 2004; 33:772-782. [PubMed: 15498744]

Wechsler, D. Wechsler intelligence scale for children, fourth edition: administration and scoring manual. San Antonio: Psychological Corporation; 2003.

Wechsler, D. Wechsler individual achievement test-2nd edition: Examiner's manual. San Antonio: Psychological Corporation; 2005.

Wehmeier PM, Schacht A, Barkley RA. Social and emotional impairment in children and adolescents with ADHD and the impact on quality of life. Journal of Adolescent Health. 2010; 46:209-217. [PubMed: 20159496]

Wender, PH. Attention deficit hyperactivity disorder in adults. USA: Oxford University Press; 1995.

Willcutt EG, Doyle AE, Nigg JT, Faraone SV, Pennington BF. Validity of the executive function theory of ADHD: meta-analytic review. Biological Psychiatry. 2005; 57:1336-1346. [PubMed: 15950006] 


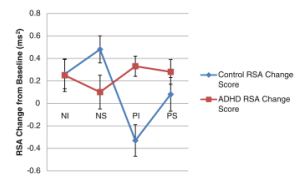

Fig. 1.

Mean change scores in respiratory sinus arrhythmia (RSA) from baseline to each of the task epochs: negative induction $(N I)$, negative suppression $(N S)$, positive induction $(P I)$, and positive suppression $(P S)$ for ADHD and control groups 
Table 1

Descriptive and diagnostic statistics for ADHD and control groups

\begin{tabular}{|c|c|c|c|c|}
\hline Variable & ADHD $(n=32)$ & Control $(n=34)$ & $F(1,65)$ & $p$ \\
\hline \multicolumn{5}{|l|}{ Demographics } \\
\hline Age & $7.91(0.69)$ & $8.12(0.69)$ & 1.60 & 0.51 \\
\hline Gender (\% male) & $62.50 \%$ & $35.30 \%$ & 5.12 & 0.03 \\
\hline Race (\% White) & $64.71 \%$ & $70.97 \%$ & 0.28 & 0.60 \\
\hline Fam. Income (\$) & $87,670(42,840)$ & $87,650(46,710)$ & 0.01 & 0.99 \\
\hline WISC-IV $^{a}$ FSIQ $^{b}$ & $105.33(14.07)$ & $106.87(12.34)$ & 0.18 & 0.62 \\
\hline \multicolumn{5}{|l|}{ 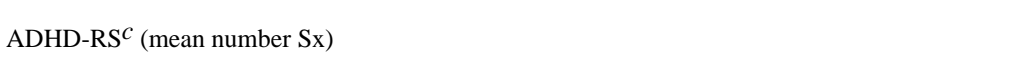 } \\
\hline Parent inattention & $6.68(1.61)$ & $4.19(1.14)$ & 49.606 & $<0.001$ \\
\hline Parent hyperactive/impulsive & $6.13(1.46)$ & $4.12(0.91)$ & 42.538 & $<0.001$ \\
\hline Teacher inattention & $6.52(0.84)$ & $3.82(0.53)$ & 123.857 & $<0.001$ \\
\hline Teacher hyperactive/impulsive & $6.40(0.96)$ & $3.87(0.43)$ & 72.587 & $<0.001$ \\
\hline \multicolumn{5}{|c|}{ Conner's 3rd edition (mean T-score) } \\
\hline Parent inattention & $73.63(10.01)$ & $49.04(9.01)$ & 105.874 & $<0.001$ \\
\hline Parent hyperactive/impulsive & $72.34(14.48)$ & $46.42(6.96)$ & 91.014 & $<0.001$ \\
\hline Teacher inattention & $68.39(9.39)$ & $46.04(6.95)$ & 145.104 & $<0.001$ \\
\hline Teacher hyperactive/impulsive & $73.00(16.51)$ & $47.82(7.17)$ & 76.267 & $<0.001$ \\
\hline \multicolumn{5}{|l|}{ Comorbid disorders $\left(\mathrm{K}^{-\mathrm{SADS}^{d}}{ }^{d}\right)$} \\
\hline Lifetime mood disorder & $0.0 \%$ & $0.0 \%$ & - & - \\
\hline Anxiety disorder & $10.0 \%$ & $5.6 \%$ & 0.60 & 0.45 \\
\hline Conduct disorder & $0.0 \%$ & $0.0 \%$ & - & - \\
\hline Oppositional disorder & $13.3 \%$ & $2.8 \%$ & 1.89 & 0.17 \\
\hline Tic disorder & $0.0 \%$ & $0.0 \%$ & - & - \\
\hline Sleep disorder & $13.3 \%$ & $2.8 \%$ & 1.89 & 0.17 \\
\hline
\end{tabular}

${ }^{a}$ WISC-IV: Wechsler Intelligence Scales for Children

$b_{\text {FSIQ: Full-Scale Intelligence Quotient (estimated) }}$

${ }^{c}$ ADHD-RS: Attention-deficit/hyperactivity disorder rating scale

${ }^{d}{ }_{\text {KSADS: Kiddie schedule of affective disorders and schizophrenia }}$ 
Table 2

Self-assessment manikin (SAM) scores across task conditions for ADHD and control groups

\begin{tabular}{lllll}
\hline Variable (mean, $\boldsymbol{S D})$ & ADHD $(\boldsymbol{n}=\mathbf{3 2})$ & Control $(\boldsymbol{n = 3 4})$ & $\boldsymbol{F}(\mathbf{1 , 6 5})$ & $\boldsymbol{p}$ \\
\hline SAM Valence/pleasure & & & & \\
Baseline 1 & $3.54(0.79)$ & $3.39(0.67)$ & 0.669 & 0.42 \\
Negative induction & $2.66(1.34)$ & $2.74(1.22)$ & 0.063 & 0.80 \\
Negative suppression & $1.76(0.82)$ & $1.91(1.18)$ & 0.326 & 0.57 \\
Baseline 2 & $3.45(0.77)$ & $3.38(0.71)$ & 0.719 & 0.40 \\
Positive induction & $4.47(1.22)$ & $4.47(0.67)$ & 0.001 & 0.99 \\
Positive suppression & $4.78(0.71)$ & $4.59(0.61)$ & 1.240 & 0.27 \\
SAM Intensity/arousal & & & & \\
Baseline 1 & $2.31(0.80)$ & $2.04(0.72)$ & 2.186 & 0.16 \\
Negative induction & $3.72(1.46)$ & $3.21(1.27)$ & 2.312 & 0.27 \\
Negative suppression & $3.25(1.63)$ & $2.85(1.28)$ & 1.221 & 0.27 \\
Baseline 2 & $2.23(0.81)$ & $1.93(0.91)$ & 0.793 & 0.38 \\
Positive induction & $2.50(1.53)$ & $2.50(1.42)$ & 0.001 & 0.99 \\
Positive suppression & $2.34(1.64)$ & $2.44(1.50)$ & 0.064 & 0.80 \\
\hline
\end{tabular}


Table 3

Respiratory sinus arrhythmia $\left(\mathrm{RSA} ; \mathrm{ms}^{2}\right.$ ) and pre-ejection period (PEP; ms) in task epochs for ADHD and control groups

\begin{tabular}{|c|c|c|c|c|}
\hline Variable (mean, $S D$ ) & $\operatorname{ADHD}(n=32)$ & Control $(n=34)$ & $F(1,65)$ & $p$ \\
\hline \multicolumn{5}{|l|}{ Baseline physiology data } \\
\hline \multicolumn{5}{|l|}{ Rest baseline } \\
\hline RSA & $7.27(1.04)$ & $7.24(1.20)$ & 0.812 & 0.37 \\
\hline PEP & $96.49(10.25)$ & $94.99(8.19)$ & 0.125 & 0.73 \\
\hline \multicolumn{5}{|l|}{ Picture baseline 1} \\
\hline RSA & $7.08(1.26)$ & $6.80(1.42)$ & 0.724 & 0.40 \\
\hline PEP & $96.59(9.15)$ & $97.79(8.05)$ & 0.320 & 0.57 \\
\hline \multicolumn{5}{|l|}{ Picture baseline 2} \\
\hline RSA & $6.95(1.16)$ & $6.97(1.41)$ & 1.80 & 0.10 \\
\hline PEP & $96.59(9.85)$ & $98.39(7.85)$ & 0.013 & 0.91 \\
\hline \multicolumn{5}{|l|}{ Task physiology data } \\
\hline \multicolumn{5}{|l|}{ Negative induction } \\
\hline RSA & $7.33(0.90)$ & $7.06(1.30)$ & 0.954 & 0.33 \\
\hline PEP & $97.31(10.09)$ & $97.33(9.37)$ & 0.001 & 0.99 \\
\hline \multicolumn{5}{|l|}{ Negative suppression } \\
\hline RSA & $7.19(0.95)$ & $7.27(1.12)$ & 0.125 & 0.73 \\
\hline PEP & $96.41(9.62)$ & $98.39(8.12)$ & 0.819 & 0.37 \\
\hline \multicolumn{5}{|l|}{ Positive induction } \\
\hline RSA & $7.28(0.97)$ & $6.64(1.27)$ & 5.163 & 0.02 \\
\hline PEP & $96.44(9.45)$ & $98.67(8.51)$ & 1.011 & 0.32 \\
\hline \multicolumn{5}{|l|}{ Positive suppression } \\
\hline RSA & $7.23(0.91)$ & $7.05(1.23)$ & 0.437 & 0.51 \\
\hline PEP & 97.37 (10.77) & $99.14(8.98)$ & 0.530 & 0.47 \\
\hline \multicolumn{5}{|c|}{ Physiology change scores } \\
\hline \multicolumn{5}{|l|}{ Negative induction } \\
\hline RSA & $0.25(0.82)$ & $0.26(0.79)$ & 0.003 & 0.95 \\
\hline PEP & $0.71(6.86)$ & $-0.48(6.64)$ & 0.505 & 0.48 \\
\hline \multicolumn{5}{|l|}{ Negative suppression } \\
\hline RSA & $0.10(0.81)$ & $0.48(0.72)$ & 3.753 & 0.04 \\
\hline PEP & $-0.19(7.49)$ & $0.62(5.67)$ & 0.237 & 0.63 \\
\hline \multicolumn{5}{|l|}{ Positive induction } \\
\hline RSA & $0.33(0.56)$ & $-0.33(0.80)$ & 14.586 & $<0.001$ \\
\hline PEP & $0.39(5.61)$ & $1.68(5.82)$ & 0.827 & 0.37 \\
\hline \multicolumn{5}{|l|}{ Positive suppression } \\
\hline RSA & $0.28(0.68)$ & $0.08(0.89)$ & 1.039 & 0.31 \\
\hline PEP & $1.31(8.17)$ & $2.12(6.08)$ & 0.206 & 0.65 \\
\hline
\end{tabular}

J Abnorm Child Psychol. Author manuscript; available in PMC 2012 August 1. 